\title{
Virtual reality headsets as external vision displays for helicopter operations: the potential of an exocentric viewpoint
}

Ernst, Johannes, Ebrecht, Lars, Schmerwitz, Sven

Johannes M. Ernst, Lars Ebrecht, Sven Schmerwitz, "Virtual reality headsets as external vision displays for helicopter operations: the potential of an exocentric viewpoint," Proc. SPIE 11019, Situation Awareness in Degraded Environments 2019, 110190J (1 May 2019); doi: 10.1117/12.2519439

Event: SPIE Defense + Commercial Sensing, 2019, Baltimore, Maryland, United States 


\title{
Virtual reality headsets as external vision displays for helicopter operations - the potential of an exocentric viewpoint
}

\author{
Johannes M. Ernst*, Lars Ebrecht, and Sven Schmerwitz \\ Institute of Flight Guidance, \\ German Aerospace Center (DLR), Braunschweig, Germany
}

\begin{abstract}
Head-worn displays (HWDs) and aircraft-mounted sensors are common means to support helicopter pilots who operate in degraded visual environment. The use of see-through HWDs is beneficial in brownout and adverse weather conditions because these displays can visualize occluded real world features like the horizon, nearby obstacles, or the desired landing spot. The German Aerospace Center (DLR) investigates an enhanced vision concept called "Virtual Cockpit". Instead of a see-through display, an immersive HWD is used to give helicopter pilots an enhanced out-the-window view. As shown in previous publications, the virtual reality (VR) technology creates benefits for several applications. This contribution explores the advantages and limitations of displaying an exocentric perspective view on the VR glasses. Moving the pilot's eye point out of the cockpit to a viewpoint behind and above the aircraft, appears to be especially useful in situations where the pilot's natural view is degraded by the own aircraft structure. Moreover, it is beneficial for certain maneuvers, in which the real location of the pilot's eye is not optimal for capturing the whole situation. The paper presents results from a simulator study with 8 participants, in which the developed symbology was tested in confined area hover and landing scenarios. The 3D exocentric perspective views increased spatial awareness in the tested scenarios and significantly reduced the required head motion. Further research is needed regarding the attitude awareness with such displays. Apart from helicopter operations, the results may also be relevant for remote piloting solutions and for other types of vehicles with restricted external vision.
\end{abstract}

Keywords: virtual reality, virtual cockpit, head-worn display, helmet-mounted display, synthetic and enhanced vision, helicopter operations, human machine interface

\section{INTRODUCTION AND RELATED WORK}

Helicopters are often operated close to the ground in the vicinity of man-made and natural obstacles like buildings, wires, or trees. To ensure safety of flight and to avoid object strikes, it is crucial for the pilots to gain and maintain comprehensive spatial awareness. However, the pilots' view of the environment is heavily restricted by non-transparent parts of the airframe. Additionally, the pilot's eyepoint is not in the best position to have a sight of the whole situation. For instance, pilots can hardly see what happens behind, above, or directly below their aircraft. This is a special form of degraded visual environment (DVE) besides the classic brownout landings and adverse weather flights.

Several state-of-the-art approaches for such problems exist. Often, crew members monitor the side- and backwards surroundings and assist the pilot verbally during confined area operations. This can be error-prone and generate great workload. In modern helicopters, databases and sensor systems with a horizontal field of view (FOV) up to $360^{\circ}$ can provide obstacle information. Examples of such short-range detection systems are the rotorstrike alerting system (RSAS) by Eurocopter ${ }^{1}$ (now Airbus Helicopters) and the obstacle proximity lidar system (OPLS) by Augusta Westland ${ }^{2}$ (now Leonardo).

After fusing data from the various sources, one typically has two choices for presenting this information to the pilot: via panel-mounted display $(\mathrm{PMD})^{3}$ or via see-through head-worn display (HWD), ${ }^{4-6}$ also known as

\footnotetext{
* johannes.ernst@dlr.de
}

Situation Awareness in Degraded Environments 2019, edited by John N. Sanders-Reed, Jarvis J. Arthur III, Proc. of SPIE Vol. 11019, 110190J - (C) 2019 SPIE · CCC code: 0277-786X/19/\$18 · doi: 10.1117/12.2519439 
helmet-mounted display (HMD). Both existing display types have their advantages and can greatly improve flight safety. Nevertheless, they also come with several limitations and weaknesses, which is why we propose to use a third display method: a non-see-through, immersive HWD, often referred to as virtual reality (VR) goggles. We call this concept Virtual Cockpit (VC).

This paper presents the development and evaluation of various 3D perspective views within the VC. Besides a transparent cockpit view where the pilot can "look through" the airframe, we show display variants with exocentric viewpoint. Such perspectives take the pilot's eyepoint to a position outside of the ownship in order to generate an overview of the situation. Such an approach was previously evaluated for fixed-wing, synthetic vision primary flight and navigation displays. ${ }^{7,8}$ Besides the different application domain, the cited projects used a PMD instead of a non-see-through HWD to show the perspective views.

Our concept is further explained in the subsequent Section 2. The approach is then evaluated by means of a simulator study, detailed in Section 3. The results of the study are presented in Section 4. Section 5 discusses the results, draws conclusions, and outlines future research directions.

\section{VIRTUAL COCKPIT WITH 3D PERSPECTIVE VIEWS}

Like traditional synthetic, enhanced, and combined vision systems, the Virtual Cockpit uses databases and aircraft-mounted sensors to generate an enhanced view of the environment under DVE conditions. The difference, however, is the non-see-through HWD that is used as display device instead of the conventional PMDs or transparent HWDs. We chose this display medium as it is expected to combine advantages of existing solutions while overcoming several weaknesses of them. For instance, an enhanced out-the-window view can be presented in a visual conformal way, ${ }^{9}$ which is not possible on a PMD. This means that the pilots can look around in the computer-generated environment by turning their heads like they are used to from flying in good visual environment. A PMD can only show a small, downscaled projection of such a view. Compared to conformal symbology on a see-through HWD, its non-see-through counterpart is free of adverse visual interferences with the real environment. For example, a full-color image with high contrast - like on a PMD - can be displayed independent of the background color and luminance conditions.

Another important plus of our VC setup is that we can create an unobstructed virtual out-the-window view. In other words, the pilots can see the surroundings in line-of-sight directions where no real cockpit windows exist or even in embedded cockpits without direct out-the-window view (armored vehicles, future supersonic aircraft). Further, the viewpoint of the synthetic view can be modified to create exocentric 3D perspective views. A detailed description of our VC concept can be found in Ref. 10. Implementation details, a discussion of limitations, and results from previous studies are described in Ref. 11.

Here, the focus is placed on the development of enhanced 3D perspective views that should improve the pilot's spatial awareness during DVE operations. As depicted in Figure 1, we implemented four display variants to be shown on the immersive HWD. Two conditions, namely Cockpit-Base and Cockpit-Trans, show the situation from a cockpit viewpoint, which reflects the normal eyepoint of a pilot sitting in the cockpit seat. In the other two conditions - Exocentric-Base and Exocentric-Trans - the observer position is moved to an exocentric viewpoint behind and above the own helicopter. Cockpit-Base represents a conventional cockpit acting as experiment baseline. Cockpit-Trans renders a semi-transparent representation of the fuselage, which allows the pilot to see through the airframe but still offers a visual reference of the ownship orientation and dimensions. Both exocentric views show the same "chase cam view". The only difference between these variants is the helicopter being transparent in Exocentric-Trans. Every display condition includes a standard primary flight display (PFD) as the only cockpit instrument. In the cockpit views, the PFD is located at its conventional position in the dashboard. The exocentric variants depict it as virtual instrument placed left of the helicopter.

All but the baseline condition Cockpit-Base incorporate visual conformal symbology. This type of symbology is well-known from augmented reality. ${ }^{9}$ The display elements - for instance a tunnel in the sky - are drawn to visually integrate with the real world as if they were part of it. Here, we use this method to increase the information content and interpretability of the 3D perspective views. The bare perspective views come with a problem called line of sight ambiguity, ${ }^{8}$ which means in this case that the helicopter position relative to other objects can hardly be determined exactly. To overcome this issue, a dropline pointing perpendicular from the 

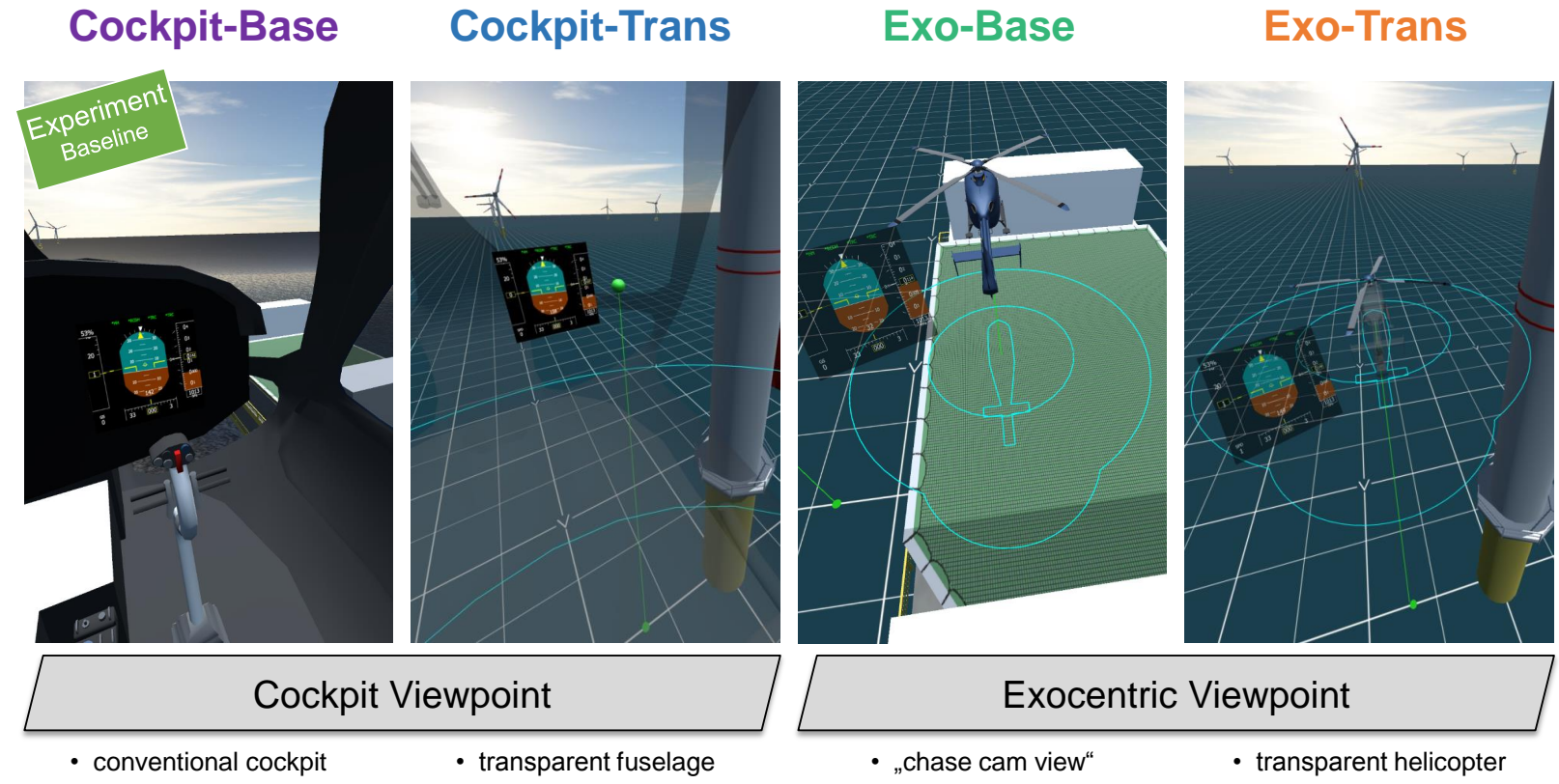

Figure 1 - The four tested perspective view types during various phases of the hover and landing scenario. Visual conformal symbology enhances Cockpit-Trans, Exocentric-Base, and Exocentric-Trans.

helicopter down to the ground is displayed. As can be seen on the two right screenshots of Fig. 1, this allows the pilot to precisely judge the aircraft position over ground. Additionally, the helicopter dimensions are projected onto the ground (or a set target height) by means of cyan outlines. A safety margin line drawn around these outlines in a distance of half a rotor diameter helps the pilot assessing obstacle clearance.

Green balls and dots on the ground are shown to indicate target positions. As shown by the Cockpit-Trans and Exocentric-Trans screenshots in Fig. 1, they can depict the desired hover position. Finally, the ocean surface in the non-baseline conditions is represented by a regular grid. It is oriented with the wind direction and indicates the wind strength by arrows rendered at the intersection points. This symbol set was developed during a previous project. $^{12}$

\section{STUDY METHOD}

To evaluate the developed 3D perspective view display, we performed a human factors study in our XR flight simulator. This section describes the simulation environment, the experimental design and the flying tasks as well as the pilots participating.

\subsection{Participants}

Eight male subjects (mean age: 39) participated in the experiment. All subjects either hold a helicopter license (1 ATPL, 2 CPL, 1 PPL) or had extensive experience with our helicopter simulator and its highly augmented flight control system and hold a fixed-wing license. The mean flight experience was $941 \mathrm{~h}$ (range: $200 \mathrm{~h}$ to $3100 \mathrm{~h}$ ). Six participants used VR glasses before and three subjects had a mean in-flight experience of $30 \mathrm{~h}$ with HWDs.

\subsection{Apparatus}

The experiment was conducted in our XR simulator. It comprises a standard aircraft cockpit with active force feedback flight controls as well as head-worn AR and VR displays. In this study, the 3D perspective views (Section 2) were displayed on the Oculus Rift CV 1. These consumer electronics VR goggles offer about $110^{\circ}$ diagonal FOV. The stereo image is created by two OLED image sources showing $1080 \times 1200$ pixels per eye. The integrated head-tracking applies a head-worn inertial measurement unit (IMU) and an external optical tracking 
unit mounted onto the cockpit structure. The image was rendered by a workstation PC equipped with an NVIDIA GeForce GTX 1070 video card and transferred via HDMI. Figure 2 shows this simulation environment. More information about the simulator can be found in Ref. 11.

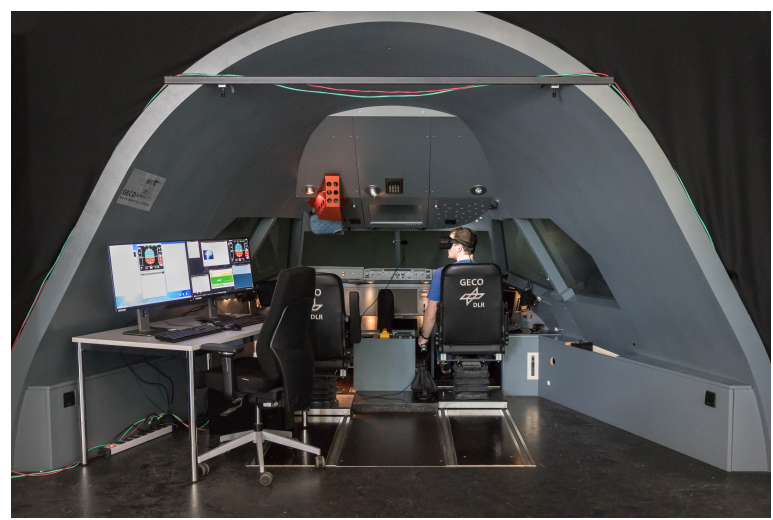

Figure 2 - The XR simulator setup with active force feedback helicopter controls and the pilot wearing non-see-through VR goggles.

To place the focus on the spatial perception task we chose a helicopter flight simulation that was easy to fly but also required permanent attention and control inputs to complete the mission. We used our in-house EC135 model with a highly augmented flight control system and various upper modes. ${ }^{13}$ This so-called command model decouples the input axes. On the lateral and longitudinal cyclic axis the "attitude command, attitude hold"-mode was applied. This implies that the pilots can directly command an attitude angle via the cyclic stick, which is hold constant until commanded otherwise. Regarding the collective we used "vertical speed command, height hold" meaning that a sink or climb rate is specified through the collective. If the collective remains in the neutral position, the aircraft remains at its current height regardless of any cyclic inputs. Finally, the pedal input axis was in "rate command, heading hold". This leads to a constant heading if the pilot does not request a turn rate via pedal inputs. To increase workload, we simulated gusty wind that was not compensated by the flight control systems. The simulated wind consisted of a constant 15 knots head-wind combined with gust components, which permanently varied between \pm 5 knots in wind direction and \pm 2.5 knots in crosswind direction. By doing so, we forced the subjects to continually monitor their aircraft state and react accordingly.

\subsection{Task}

The experiment was split into two separate scenarios, which are explained in the following subsections. Both use cases are tasks performed by helicopter crews operating in offshore windparks.

\subsubsection{Hovering next to wind turbine tower}

The first task was a hover maneuver next to a wind turbine tower. This maneuver is used to hoist persons or freight to and from the lower access platform of offshore wind turbines. To do so, the pilot has to steer the helicopter to a position abeam the tower. Thereby, the clearance between the rotor tips and the wind turbine is required to be close to the minimum safety distance of $5.1 \mathrm{~m}$ (half of rotor diameter) while not going below it. Figure 3a shows a helicopter in the desired position.

In our experiment the participants flew a straight approach starting $0.25 \mathrm{NM}$ out and $250 \mathrm{ft}$ above the target hover position. The initial airspeed was 40 knots with 15 knots headwind. The pilots were instructed to acknowledge when in the desired position by pushing a button on the cyclic stick. This started a 2-min hover phase in which the subjects should hold the desired position as precisely as possible. Two of the screenshots in Fig. 1 show this maneuver while approaching the wind turbine (Cockpit-Trans) and during the hover phase (Exocentric-Trans). 


\subsubsection{Landing on offshore platform between obstacles}

The second task was a confined area landing on an offshore platform. The approach phase was similar to the hover task: straight towards the wind, $0.25 \mathrm{NM}$ out and $250 \mathrm{ft}$ above the helipad with an initial airspeed of 40 knots. The whole maneuver had to be conducted with head-wind. As the direct approach path was blocked by an obstacle on the platform (see Fig. 3b), the pilots had to approach a point on the left of the helipad and then hover sideways to the right to reach the desired landing spot. Figure 3b shows a helicopter with the desired "headwind-heading" in its final position in the middle between the two obstructions. The width of the landing pad was $22.4 \mathrm{~m}$, the clear space between the obstacles was $31.3 \mathrm{~m}$.

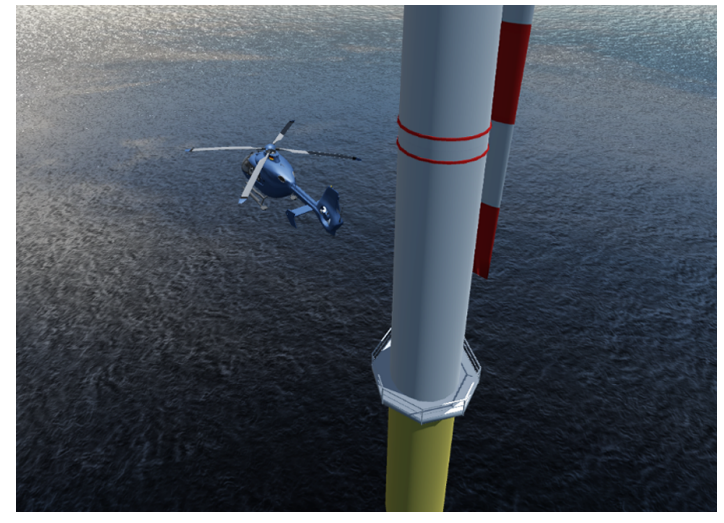

(a) The helicopter in the desired hover position abeam the wind turbine tower.

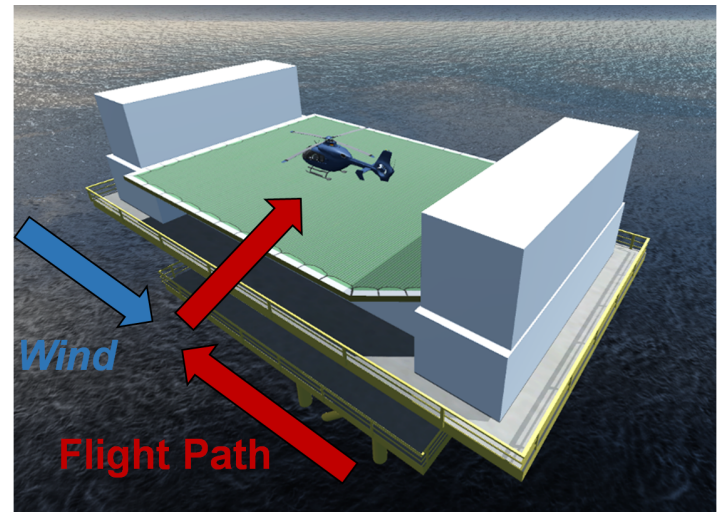

(b) The helicopter in the desired landing position in the middle of the offshore platform.

Figure 3 - Illustration of the hover and the landing scenario.

\subsection{Experimental Design}

After briefing and biographical questionnaire, the subjects started the experiment with a training session. During that, both mission tasks were flown with each display variant until the subject was familiar with the simulator and the symbology. For the following experiment, a within-subject design was applied. This means that every participant tested every display condition. The testing phase was split into two blocks separated by a 15-min break. Each block lasted approximately $40 \mathrm{~min}$ and comprised all runs with two selected display variants: either both cockpit views or both exocentric views. All runs with one display condition were flown in a row. The subjects performed two landings and two hover maneuvers per display variant. The condition order was counterbalanced between subjects. After the testing phase, the participants were asked to provide subjective feedback via a debriefing questionnaire. The total experiment duration was about $3 \mathrm{~h}$.

\section{STUDY RESULTS}

This section describes our major findings concerning the effects of the developed view types on spatial awareness, head motion, and helicopter attitude perception.

\subsection{Spatial Awareness}

The tested 3D perspective views were developed to improve the pilots' perception of the surroundings in confined area operations. This section analyzes if the displays really could improve spatial awareness. To do so, subjective pilot feedback and several flight performance measures are presented.

\subsubsection{Flight performance - hover task}

Regarding the hover task, we were interested in how well the pilots could hold the helicopter in the desired hover zone left of the wind turbine tower. A circular area $2.5 \mathrm{~m}$ around the target position was defined as the "desired" zone. Aircraft positions outside of that zone but still within a $5 \mathrm{~m}$ radius were considered "adequate". Farther 
deviations were classified as "out of bound". The evaluation reveals clear advantages for both exocentric views over the cockpit perspectives. Using Cockpit-Base the participants stayed only $11 \%$ of the hover duration within the "desired" limits and $52 \%$ "out of bound". Cockpit-Trans produced time portions of $26 \%$ in "desired" and $35 \%$ in "out of bound". This performance was significantly improved by Exocentric-Base and Exocentric-Trans where the pilots hovered only $6 \%$ and $11 \%$ of the time "out of bound". The corresponding values for the "desired" zone were $51 \%$ and $46 \%$ respectively. ${ }^{11,14}$

\subsubsection{Flight performance - landing task}

In the landing scenario, the pilots were instructed to approach an offshore platform from the left side and land the aircraft sideways between two obstacles. The task was to leave sufficient obstacle clearance during the whole maneuver and touch down in the middle of the platform.

Figure 4 shows top views of the touchdown positions achieved with the four display conditions. The comparison reveals that the lateral position deviation was higher with Cockpit-Base (median: $1.3 \mathrm{~m}$ ) compared to CockpitTrans (median: 0.8 m), Exocentric-Base (median: $0.4 \mathrm{~m}$ ) and Exocentric-Trans (median: 0.5 m). Furthermore, the measured deviations are spread wider with the cockpit variants. The median longitudinal deviations were within every condition - larger than the corresponding lateral offsets. They range from $1.3 \mathrm{~m}$ (Exocentric-Trans) via $1.2 \mathrm{~m}$ (Cockpit-Base) and $1.0 \mathrm{~m}$ (Cockpit-Trans) to $0.8 \mathrm{~m}$ (Exocentric-Base). Also, the values of Cockpit-Base are widely spread and the exocentric views seem to have a tendency to a touchdown position slightly ahead of the desired position.
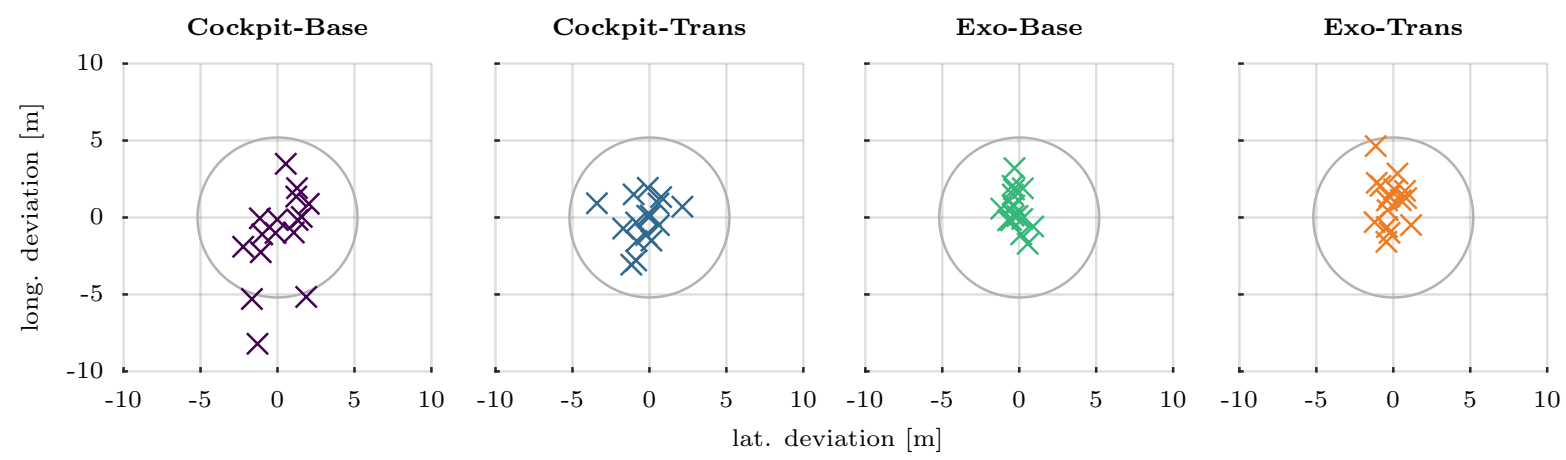

Figure 4 - Top views of the touchdown positions obtained with the four display variants. The graphs show lateral and longitudinal deviations from the desired landing spot in the middle of the platform.

To evaluate how well the pilots maintained the required obstacle clearance, three classes were defined: "desired" refers to a distance to the nearest object of more than three-fourths of the rotor diameter $(7.65 \mathrm{~m})$; "adequate" covers smaller distances that are larger than half a rotor diameter $(5.1 \mathrm{~m})$; even smaller clearances are considered "below safety limits". Figure 5 shows for how long the obstacle clearance was within each of these zones (measured in percentage of the total landing duration). As expected, the distance to the obstacles was as "desired" or at least "adequate" most of the time for all view types. However, the pilots in the conventional cockpit operated below the safety limits in $8 \%$ of the time. With Cockpit-Trans and Exocentric-Trans this portion was 4\%. Only with Exocentric-Base the time share below the safety limits was less than $1 \%$. As a side note, the duration of landing maneuver was not restricted and no significant differences between the display conditions were observed. 

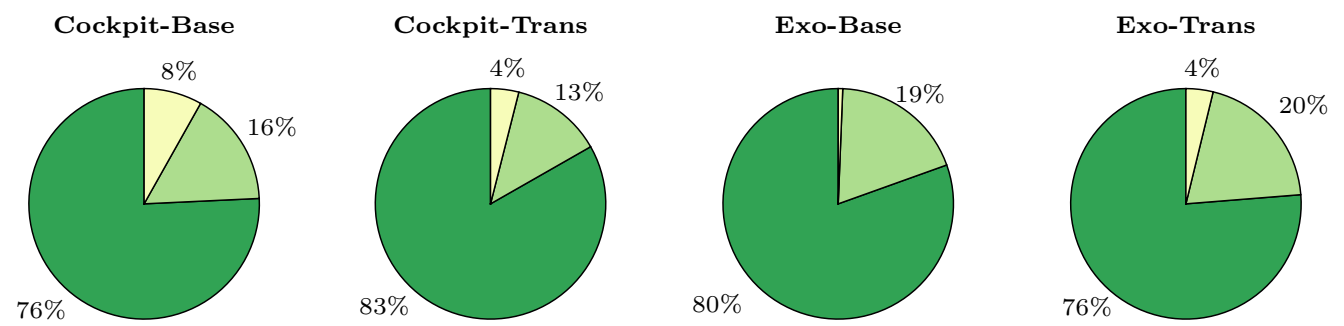

Obstacle clearance classification: $\square$ desired $(>7.65 \mathrm{~m})$ adequate $(>5.1 \mathrm{~m})$ below safety limits

Figure 5 - Obstacle clearance during the landing phase: pie charts show how long the aircraft was within the defined obstacle clearance zones, in percentages of the total landing duration.

\subsubsection{Pilot feedback}

The debriefing questionnaire confirmed the advantages of the exocentric viewpoint regarding spatial and obstacle awareness. All pilots agreed that this view "supports collision avoidance" and "improves spatial orientation". Further, the questionnaire revealed that the participants felt safer in the exocentric variants because of the obstacles being visible. Concerning the judgment of the distance to obstacles in the front, on the side, and in the back of the helicopter, the cockpit view could also not keep up with its counterpart. Despite that, the results cannot clarify whether the exocentric perspectives reduced workload. However, an increase in workload was not reported either. Additionally, the subjects acknowledged the usefulness of the visual conformal symbology. The safety margin circle, the dropline, and the target markers clearly improved the spatial awareness with Exocentric-Base and Exocentric-Trans. The pilots stated that they could adequately estimate the height above the landing pad with all variants, while the mean rating of Exocentric-Trans was slightly lower than for the other variants.

\subsection{Head Motion}

As described in Section 2, the exocentric display variants provide the pilots with a view from a position outside of the ownship. As a result, the visually observed space is different from the conventional cockpit view. For instance, the pilots are able to see obstacles on the side of their helicopter without turning their heads. Since continual head motion can increase workload and lead to fatigue, the following paragraphs illustrate how the tested display conditions affected the pilots' head rotation behavior.

\subsubsection{Hover task}

Figure 6 depicts the typical head motion behavior of a participant performing the hover task with the baseline condition Cockpit-Base. The upper curve shows that the pilot turned his head about $40^{\circ}-60^{\circ}$ to the right towards the wind turbine tower. Moreover, he switched his view to the aircraft centerline direction with fast and regular movements. During the whole hover maneuver, the head pitch/elevation angle remained within $0^{\circ}$ and $-20^{\circ}$. Apparently, the pilot tilted his head slightly downwards when he looked in the longitudinal direction of the aircraft. With Cockpit-Trans the head motion behavior looked similar in many cases. However, some participants also regularly tilted their heads downwards more than $50^{\circ}$ so as to check the position deviation via dropline and target dot symbology located under the aircraft. 

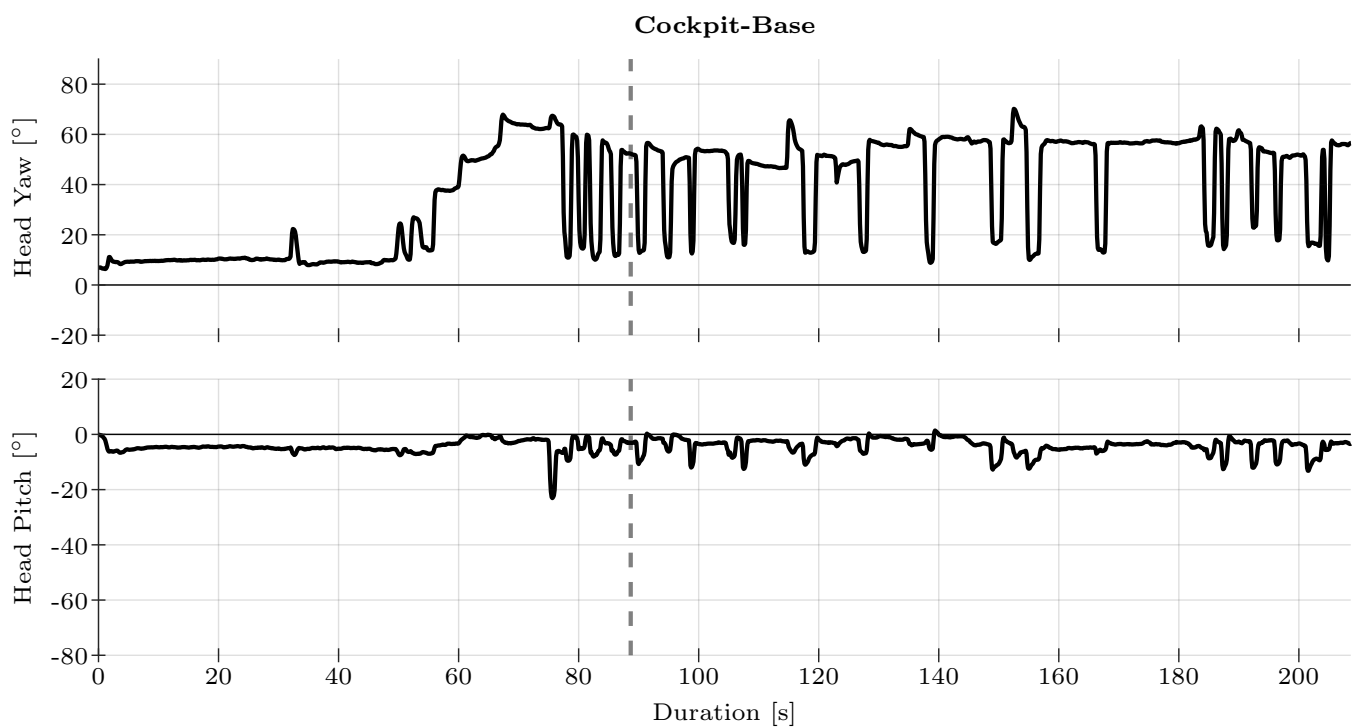

Figure 6 - Typical head motion during the hover task with Cockpit-Base. The dashed line indicates the start of the 2 min-hover-phase.

Both exocentric variants led to entirely different head motion curves. As shown by Fig. 7, the typical head azimuth and elevation curves show few movements during the approach phase and almost no head motion during the hover phase. The pilot continuously looked in forward direction with his head tilted $40^{\circ}$ down. The negative pitch attitude can be explained by the ownship and the hover position symbology being located below the viewpoint.
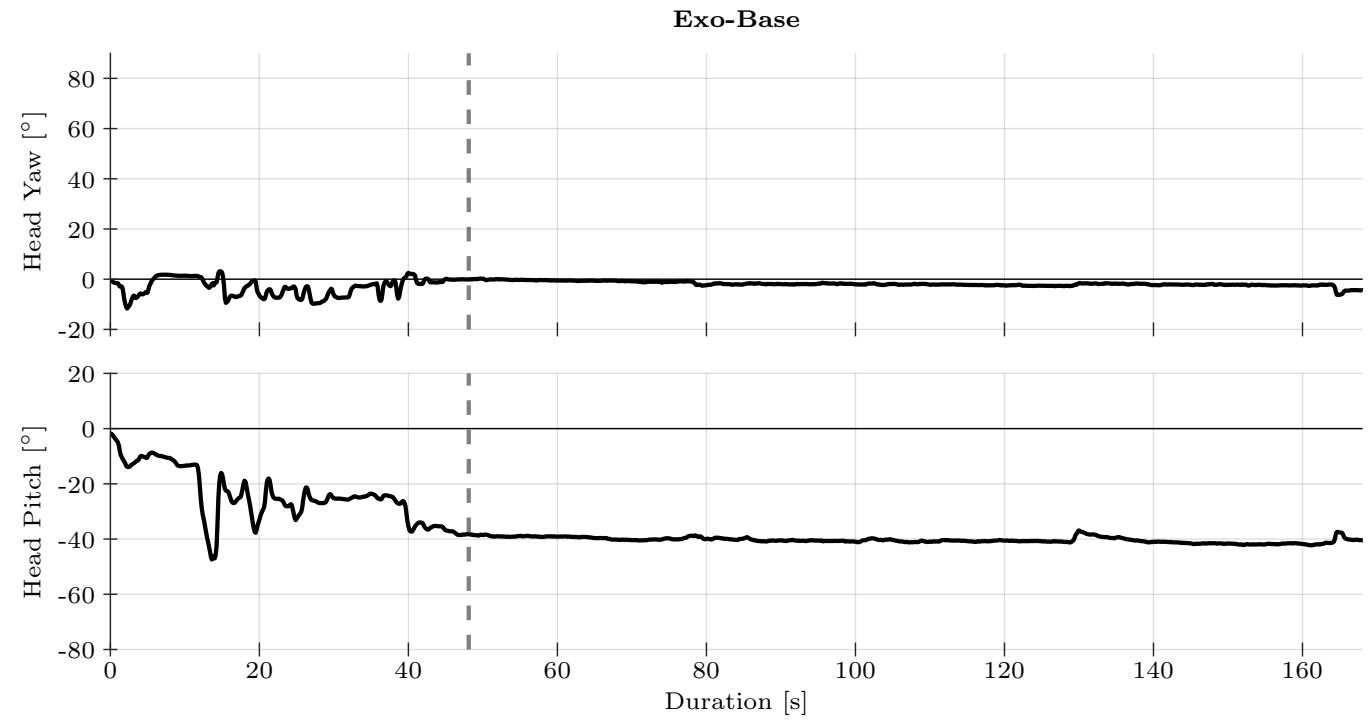

Figure 7 - Typical head motion during the hover task with Exocentric-Base. The dashed line indicates the start of the 2 min-hover-phase.

The observations from the typical head movement plots can be confirmed for all participants by evaluating how long the pilots' head rotation was above and below specific threshold angles. Rotations smaller than $20^{\circ}$ were categorized as "low" while azimuth angles between $20^{\circ}$ and $50^{\circ}$ were assigned to the "medium" group and the rest was classified as "high". Figure 8 shows the pilots' head motion during the 2 min-hover-phase grouped into the specified zones. In the exocentric conditions the pilots never turned their heads more than $20^{\circ}$ left or 
right. In contrast, the head azimuth was "high" in $80 \%$ of the hover time with Cockpit-Base. Cockpit-Trans could reduce this amount to $61 \%$.
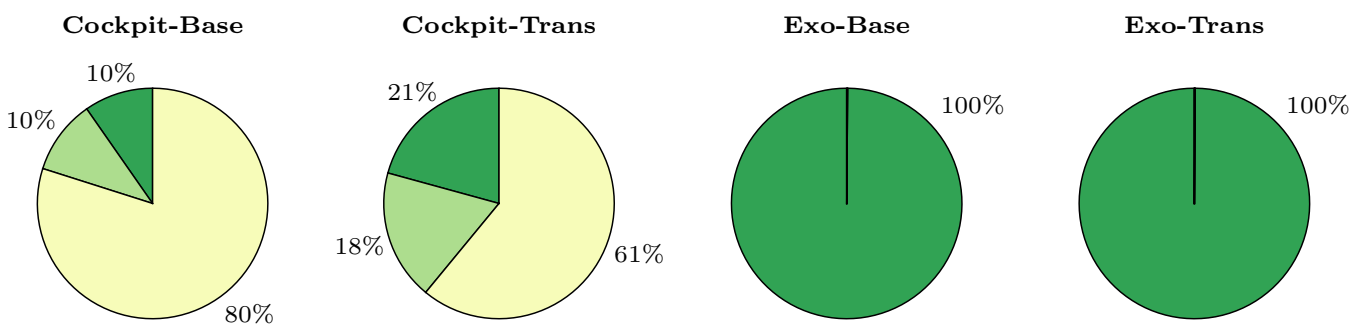

Head rotation classification:

$$
\square \text { low }\left(\varphi<20^{\circ}\right) \square \text { medium }\left(20^{\circ}<\varphi<50^{\circ}\right) \square \text { high }\left(\varphi>50^{\circ}\right)
$$

Figure 8 - Head azimuth rotation $\varphi$ during the hover task: pie charts show how long the pilots' head azimuth angle was within the specified rotation classes, in percentages of the total hover duration.

Regarding the elevation rotation, both exocentric views caused the pilots to tilt their heads downwards between $20^{\circ}$ and $50^{\circ}$ in $85 \%$ of the time. In contrast, when the pilots flew with the cockpit views, their head elevation rotation was within $\pm 20^{\circ}$ in $89 \%$ (Cockpit-Base) and $76 \%$ (Cockpit-Trans) of the hover time . Cockpit-Trans was the only display variant where head elevation angles larger than $50^{\circ}$ were observed $(7 \%)$. This can be explained by the pilots using the dropline and the target dot under the aircraft for position holding.

Finally, the total angular distance covered through head rotations during the hover phase was computed. In azimuth direction, the median total rotation was $996^{\circ}$ for Cockpit-Base and $1430^{\circ}$ for Cockpit-Trans. In contrast, the pilots turned their heads only $99^{\circ}$ with Exocentric-Base and $117^{\circ}$ with Exocentric-Trans. Regarding the head elevation rotation, the median of the total covered angular distance was highest with Cockpit-Trans $\left(485^{\circ}\right)$ followed by Cockpit-Base $\left(291^{\circ}\right)$, Exocentric-Trans $\left(209^{\circ}\right)$, and Exocentric-Base $\left(150^{\circ}\right)$.

\subsubsection{Landing task}

During the landing task, mostly similar head motion behavior was observed. Nevertheless, due to the different task characteristics, the head motion was not as extensive as during the hover task. Again, with Cockpit-Base the pilots quickly turned their head between forward and rightward direction in order to land the helicopter sideways between the obstacles. Typically, Cockpit-Trans caused large downward tilts when the participants used the dropline to judge their position over ground. Similar to the hover task, both exocentric views required no fast line of sight changes.

Figure 9 shows that the azimuth rotation was smaller than $20^{\circ}$ in $98 \%$ of the time flying in the exocentric conditions. Cockpit-Base and Cockpit-Trans provoked azimuth rotations larger than $50^{\circ}$ in about one-fifth of the time. The durations in the specified elevation rotation zones were comparable to the values observed during the hover task. Only Cockpit-Trans caused head tilts higher than $50^{\circ}$ downwards (4\%). With the exocentric viewpoints, the pilots tilted their heads down by $20^{\circ}$ to $50^{\circ}$ for $80 \%$ of the landing duration. By contrast, with the conventional view Cockpit-Base the vertical line of sight direction was within $\pm 20^{\circ}$ for the same amount of time. With the transparent cockpit this value was reduced to $55 \%$. 

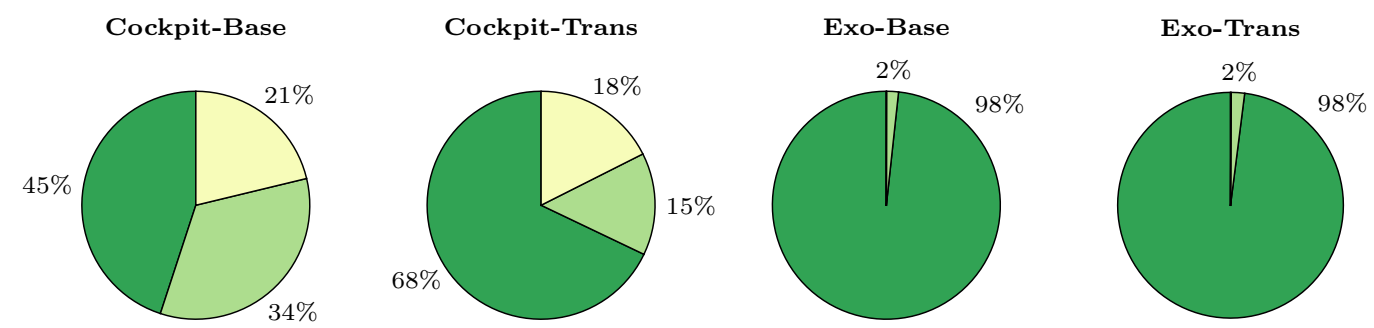

Head rotation classification:

$\square$ low $\left(\varphi<20^{\circ}\right)$

$\square$ medium $\left(20^{\circ}<\varphi<50^{\circ}\right)$

$\operatorname{high}\left(\varphi>50^{\circ}\right)$

Figure 9 - Head azimuth rotation $\varphi$ during the landing task: pie charts show how long the pilots' head azimuth angle was within the specified rotation classes, in percentages of the total landing duration.

The median angular distance that the pilots covered by turning their heads in azimuth direction was highest with Cockpit-Base $\left(879^{\circ}\right)$, followed by Cockpit-Trans $\left(742^{\circ}\right)$. Similar to the hover task, significantly lower head turn medians of $84^{\circ}$ and $110^{\circ}$ were measured for Exocentric-Base and Exocentric-Trans. The angular distances in elevation direction were $282^{\circ}$ for Cockpit-Trans, $123^{\circ}$ for Cockpit-Base, $110^{\circ}$ for Exocentric-Trans, and $71^{\circ}$ for Exocentric-Base.

\subsection{Helicopter Attitude Perception and Control}

One important research question of this study was if the pilots would be able to control the helicopter from a viewpoint outside of the cockpit. The experiment confirmed that all subjects could perform their tasks with the developed exocentric views. Nevertheless, the pilots also reported that controlling the attitude of the helicopter appeared to be easier when sitting inside the cockpit. Further, a few participants stated that they had to use the PFD more often to assess the attitude when flying with the exocentric views.

We explored this issue by comparing the distributions of pitch angles measured for each of the four view types. Figure 10 depicts boxplots of these distributions for hover and landing task. As expected, the medians do not significantly differ. However, the boxplots, which are based on eight pitch measurements per second, reveal that the distribution width for Cockpit-Base is smaller than for all non-conventional display conditions. Especially the boxplot of Exocentric-Trans for the hover task has a wider interquartile range (IQR) and outliers ranging from $-20^{\circ}$ to $28^{\circ}$. In summary, the boxplots show that the pilots commanded more extreme pitch angles with the transparent cockpit and both exocentric views. Nevertheless, it has to be noted that these large values occurred rarely and differences of the middle $50 \%$ (IQR) of the data are relatively small.

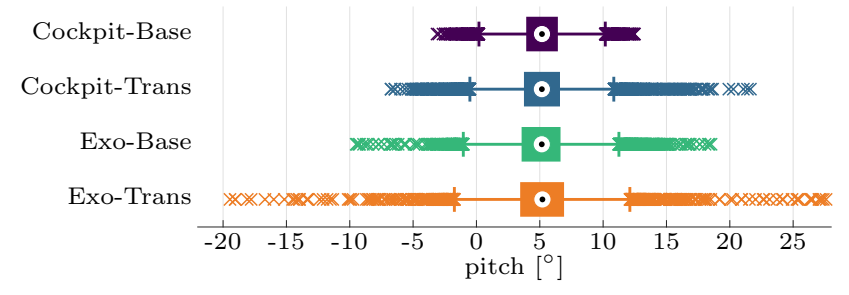

(a) Hover task.

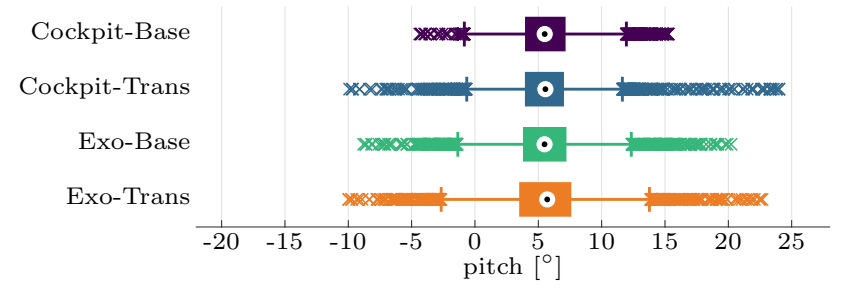

(b) Landing Task.

Figure 10 - Distribution of recorded pitch angles during both maneuvers. Boxplots show median (dot/circle), 25th and 75th percentiles (filled rectangle), and outliers (x markers) with whisker length 1.5 IQR. 


\section{DISCUSSION, CONCLUSION AND FUTURE WORK}

The study was conducted to find out if the developed 3D perspective view can improve the spatial awareness and flight performance of helicopter pilots during confined area operations. Further, we wanted to investigate if it is possible to control the helicopter with these views.

In summary, we received positive pilot feedback on the evaluated concept. The hover scenario revealed clear advantages for the exocentric views over the conventional cockpit view presented on the VR-HWD. Compared to the conventional flight deck, the transparent cockpit with conformal symbology had advantages concerning spatial awareness. Nevertheless, it was clearly outperformed by the exocentric viewpoints. Even though Cockpit-Trans displayed the desired hover position by means of the conformal overlay, the pilots could not translate this information to better performance. In our opinion, this is mainly caused by the fact that it requires a lot of effort to visually gather all required information: the wind turbine at the right, the PFD on the dashboard straight ahead, the target dot below, et cetera. In contrast, the exocentric views present an overview of the whole situation with all information available within a small area of the forward FOV. In the landing scenario, the flight performance differences between the display conditions were not as large as during the hover maneuver but still visible. In total, Exocentric-Base performed best as it caused the smallest deviations from the desired touchdown position and also prevented the pilots from flying to close to the obstacles. Especially the improved awareness of the obstacle clearance during confined area operations appeared as a major plus for this display variant.

Regarding the participants' head motion, we found clear differences between the ego- and the exocentric viewpoint conditions. The tested maneuvers caused the pilots to have their heads turned to the right for a significant amount of time when sitting inside the cockpit. Furthermore, we saw many fast line-of-sight switches between the forward direction and the rightward direction where the wind turbine tower or the landing pad was located respectively. Of course, these phenomena are highly task-dependent. In this study they were more pronounced in the hover scenario than in the landing task. With the exocentric perspective, the participants did not have to turn their heads. As expected, they just kept their heads tilted slightly downwards to have the helicopter and the dropline in sight. We observed two strategies to mitigate this issue. First, many subjects did not hover directly abeam the wind turbine so as to have the tower at 1 or 2 o'clock instead of the desired 3 o'clock position. Second, some pilots turned the aircraft nose out of the wind towards the obstacle. As the peripheral vision is restricted by the limited FOV of the VR goggles, future work should also research if a larger FOV has an influence on the head motion characteristics. Fewer head movements and less strenuous head poses appear to be a clear advantage concerning workload and fatigue. Nevertheless, it is also important to note that head motion can also have positive effects on creating spatial awareness.

A weakness of all non-conventional 3D perspective views seems to be that they prompted the pilots to command higher maximum and lower minimum pitch angles. An explanation for this can be the missing or less apparent visual cues for attitude perception. When sitting inside the cockpit, the motion of the airframe relative to the external scene is an important indication for a pilot giving him an instant impression of the helicopter attitude. The horizon moving relative to the instrument panel is even perceived via peripheral vision when the pilot is focusing on another task. The semi-transparent airframe of Cockpit-Trans makes this cue less striking and the exocentric viewpoint does not offer it at all. This is in line with some subjects reporting that more frequent usage of the PFD was required when flying the exocentric view conditions. An inferior reason for the wider pitch angle distribution can also be that even small position deviations are easily seen with the conformal symbology. As a consequence, the pilots might have tried to more rapidly correct that with larger control inputs.

In conclusion, the study proved the capabilities of 3D exocentric perspective views displayed in the Virtual Cockpit. The perception of nearby obstacles and the overall spatial awareness appeared to be significantly improved in comparison with a conventional cockpit view on a non-see-through HWD. The next steps in this project focus now on enhancing the exocentric symbology. For instance, we are testing several concepts to improve the attitude perception. Moreover, we are currently integrating the VR systems into our motion simulator in order to investigate the influence of motion cues in exocentric views. Thereafter, flight tests and a comparison with a state-of-the-art symbology on a see-through HWD are planned. Besides the described helicopter operations, the concept can also be tested for embedded flight decks or other cockpits with restricted window areas. Examples include armored military vehicles and supersonic airplanes. 


\section{REFERENCES}

[1] Waanders, T., Scheiblhofer, R., Qian, Q., van Noort, B., Ziegler, V., Schubert, F., and Koerber, R., "Helicopter rotorstrike alerting system," Proc. 41st European Rotorcraft Forum (2015).

[2] Brunetti, M., "The Guardian project: Reasons, concept and advantages of a novel obstacle proximity LIDAR system," Proc. 40th European Rotorcraft Forum (2014).

[3] Szoboszlay, Z. P., Fujizawa, B. T., Ott, C. R., Savage, J. C., Goodrich, S. M., McKinley, R. A., and Soukup, J. R., "3D-LZ Flight Test of 2013: Landing an EH-60L Helicopter in a Brownout Degraded Visual Environment," Proc. 70th American Helicopter Society International Annual Forum (2014).

[4] Völschow, P., Münsterer, T., Strobel, M., and Kuhn, M., "Display of real-time 3D sensor data in a DVE system," Proc. SPIE, 98390N (2016).

[5] Viertler, F. and Hajek, M., "Evaluation of Visual Augmentation Methods for Rotorcraft Pilots in Degraded Visual Environments," Journal of the American Helicopter Society 62(1), 012005 (2017).

[6] Schmerwitz, S., Lüken, T., Döhler, H.-U., Peinecke, N., Ernst, J. M., and da Silva Rosa, D., "Conformal displays: human factors analysis of innovative landing aids," Optical Engineering 56(5), 051407 (2017).

[7] Ebrecht, L. and Schmerwitz, S., "Integration of a 3D perspective view in the navigation display: featuring pilot's mental model," Proc. SPIE 9471, 94710C (2015).

[8] Wickens, C. D., "Aviation Displays," in [Principles and practice of aviation psychology], Tsang, P. S. and Vidulich, M. A., eds., 147-200 (2003).

[9] Peinecke, N., Schmerwitz, S., Döhler, H.-U., and Lüken, T., "Review of conformal displays: more than a highway in the sky," Optical Engineering 56(5), 051406 (2017).

[10] Ernst, J. M., Döhler, H.-U., and Schmerwitz, S., "A concept for a virtual flight deck shown on an HMD," Proc. SPIE 9839, 983909 (2016).

[11] Ernst, J. M., Peinecke, N., Ebrecht, L., Schmerwitz, S., and Döhler, H.-U., "Virtual Cockpit: an immersive head-worn display as human-machine interface for helicopter operations," Optical Engineering 58(5), 051807 (2019).

[12] Ernst, J. M., Ebrecht, L., and Erdmann, S., "Synthetic vision on a head-worn display supporting helicopter offshore operations," Proc. SPIE 10642, 106420B (2018).

[13] Greiser, S., Lantzsch, R., Wolfram, J., Wartmann, J., Müllhäuser, M., Lüken, T., Döhler, H.-U., and Peinecke, N., "Results of the pilot assistance system 'Assisted Low-Level Flight and Landing on Unprepared Landing Sites' obtained with the ACT/FHS research rotorcraft," Aerospace Science and Technology 45, 215-227 (2015).

[14] Ernst, J. M., "3D perspective views on immersive head-worn displays: Can exocentric views increase pilot situational awareness?," Proc. 31st Congress of the International Council of the Aeronautical Sciences (ICAS) (2018). 